\title{
Modern human behaviors during the late stage of the MIS3 and the broad spectrum revolution: Evidence from a Shuidonggou Late Paleolithic site
}

\author{
GUAN Ying ${ }^{1}$, GAO Xing ${ }^{1 *}$, LI Feng $^{1,2}$, PEI ShuWen ${ }^{1}$, CHEN FuYou $^{1} \&$ ZHOU ZhenYu ${ }^{1,3}$ \\ ${ }^{1}$ Laboratory of Human Evolution, the Institute of Vertebrate Paleontology and Paleoanthropology, Chinese Academy of Sciences, Beijing 100044, \\ China; \\ ${ }^{2}$ Graduate University of Chinese Academy of Sciences, Beijing 100049, China; \\ ${ }^{3}$ Institute of Archaeology, Chinese Academy of Social Sciences, Beijing 100710, China
}

Received July 27, 2011; accepted September 13, 2011; published online November 12, 2011

\begin{abstract}
The last glacial period was vital for the distribution and evolution of early modern humans in Asia. The Shuidonggou Late Paleolithic site, dated at 30-20 ka BP, accumulated cultural remains during the important late stage of MIS 3 period in the last glacial. These remains represent characteristics of typical Late Paleolithic conditions in North China: high degree of standardization and morphological variability of tool types, exploitation of bone materials, systematic use of body decorations, extensive use of earth-pit hearths, distinct functional spatial organization within habitations, and conversion of subsistence patterns. These characteristics illustrate early modern human behaviors during the late MIS3 period, and provide clues and perspectives for the analysis of early modern human origins in China. At the same time, the conversion of subsistence patterns is considered to be a combination of multiple early modern human behaviors, as well as the result of the Broad Spectrum Revolution. In this paper, we argue for the dynamic mechanism of Broad Spectrum Revolution from a human behavioral and ecological perspective.
\end{abstract}

Late Paleolithic, early modern human behavior, broad spectrum revolution, environmental change

Citation: Guan Y, Gao X, Li F, et al. Modern human behaviors during the late stage of the MIS3 and the broad spectrum revolution: Evidence from a Shuidonggou Late Paleolithic site. Chin Sci Bull, 2012, 57: 379-386, doi: 10.1007/s11434-011-4828-x

During the last stage of the Pleistocene, Homo sapiens sapiens proliferated globally and eventually occupied each continent, except Antarctica. This expansion established a rich and colorful Upper Paleolithic culture [1-4]. In China, Paleolithic cultures in the north were dominated by the Small Flake Tool Cultural Complex, and in the south by the Pebble Tool Cultural Complex [5-7]. In addition, Levallois, blade and microblade technologies co-existed with these two main cultural complexes. Together, this composite formed the unique paleolithic culture of East Asia, which differed from those in North America, Central Asia, West Asia, Europe and Africa [1,8].

During the Late Pleistocene, the natural environment

*Corresponding author (email: gaoxing@ivpp.ac.cn) fluctuated dramatically. These environmental fluctuations were associated with evolution of Paleolithic populations. The last glacial began about 100000 years ago, and culminated during the Holocene. However, there was a relative warm and wet interglacial epoch between 60000 and 20000 years ago, which contained several short time scale climatic events, and affected the flora and fauna to a global extent. These changes caused the appearance or extinction of many species in the high latitude of the Northern Hemisphere. Among these species, human beings showed the most considerable response to the environmental change, and accordingly created different kinds of Paleolithic cultures. Bar-Yosef $[9,10]$ summarized several universal characteristics of the Upper Paleolithic Revolution, based on materials from throughout the world. These materials included sys- 
tematic production of prismatic blades; a high degree of standardization and morphological variability of tool types; exploitation of bone and antler as raw materials for the production of daily or ritual tools; systematic use of grinding and pounding stone tools; systematic use of body decorations; long-distance exchange networks in lithics, raw materials, and marine shells; the invention of improved hunting tools; the creation of human and animal figurines; decorated and carved bone, antler, ivory and stone objects; the invention of food storage facilities; structured hearths with or without the use of rocks for warmth baking and food processing; distinct functional spatial organizations within habitations and hunting stations; and potential changes in subsistence activities and burials. These characteristics also are regarded as the mark of early modern human behavior, and indicate humanity's evolution to a new stage. Thus, this information can be used as objective criteria for the evaluation of human cognition capabilities.

Another equally vital research field refers to human subsistence strategies. Up until the 1960s, Flannery proposed the Broad Spectrum Revolution (BSR hereafter) hypothesis, based on the subsistence pattern changes of ancient foragers under the pressure of natural environmental changes and the expansion of human populations. This hypothesis suggested that human beings actively broadened their food resources under harsher environmental conditions, when many resources which had not been utilized before, such as wild grass seeds and mollusks, came into the human diet $[11,12]$. In addition, hunting, food processing and storage technologies, and spatial organization within habitation, all advanced and improved along with this shift [13]. With more archaeological evidence found recently, the origination time of the BSR might be speculated to be as far back as the last glacial, which is about $23 \mathrm{ka} \mathrm{BP} \mathrm{[14-16].}$

These hypotheses constitute the framework and provide a direction for Upper Paleolithic research, as is the case in this work. In this paper, we focus on stone tool technology, the intellectual level of foragers in the last glacial, and survival patterns of ancient occupants at Locality 2 of the Shuidonggou Site (SDG Loc. 2 hereafter), and we discuss the early modern human population in this area during the later stage of the MIS3. With the help of human behavioral ecology models, we also attempt to interpret the process of subsistence pattern changes and the mechanism of the BSR.

\section{Archaeological context and research materials}

SDG Loc. 2 is located in Lingwu County, Ningxia Hui Autonomous Region, about $18 \mathrm{~km}$ east of the Yellow River. The SDG site is considered to be a typical Late Paleolithic site in North China. In China, there are different cultural traditions compared to other locales, and there is no typical Middle Paleolithic cultural complex. Thus, the prehistoric time before the Holocene is divided into two stages: Early and Late Paleolithic. The Late Paleolithic to Upper Paleolithic periods in Europe and North America fall within this North China time range. Many studies on the topic have been published [17-21]. Thus, we do not elaborate on the site information in this paper. Dating results suggest that anthropogenic layers were deposited during 30-20 ka BP [20-30], which belong to the later stage of the MIS3. In terms of the site's stratigraphic profile, level 4 comprises yellow sand, with stone artifacts, animal bones and ash. Anthropogenic layer 1 (refer to as cultural layer 1) is present in stratigraphic level 4, which has a maximum thick-

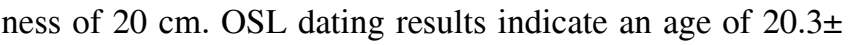
$1 \mathrm{ka} \mathrm{BP}$. Level 6 is characterized by yellow sand, with stone artifacts, animal bones and ash. Anthropogenic layer 2 (refer to as cultural layer 2) is present in level 6 , which has a maximum thickness of $30 \mathrm{~cm}$. OSL dating and $\mathrm{AMS}^{14} \mathrm{C}$ results indicate an age range of $28-25 \mathrm{ka} \mathrm{BP}$. These two levels have the largest excavation area, and yielded the most unearthed specimens and relics.

\section{Evidence of early modern human behavior}

Tens of thousand of specimens, including stone artifacts, animal bones and ornaments were unearthed in cultural layers 1 and 2. This material also included eight earth-pit hearths. These specimens and relics provide reliable evidence for evaluating the property of SDG Loc. 2. Six characteristics were extracted as distinctive indicators of Upper Paleolithic culture and early modern human behavior:

(1) High degree of standardization and morphological variability of tool types. In SDG Loc. 2, the stone tool assemblage includes scrapers, end scrapers, points, drills, burins, and a few chopping tools. Except for the end scraper, the other tool types are present in earlier Paleolithic sites in China. However, elsewhere these are not as sophisticated and standard as they are at SDG Loc. 2. In addition, the variety of tool types reflects a high degree of morphological variability. For example, the end scraper is made of a long flake with ridges on the dorsal surface, and was retouched mostly on one end of the ventral to dorsal surface, which resulted in long and deep scars. The edge angles normally are not sharp $\left(\geqslant 60^{\circ}\right)$. The edge was repaired to the shape of an arc. The raw material for the end scraper is chert (Figure $1(1-4))$. With regard to the scraper, there also are highly standard sub-types, such as the converged type and the straight end edge type (Figure 1(5-10)). The raw materials are dominated by chert, followed by dolomite and some other materials.

(2) Exploitation of bone materials. One bone needle was unearthed near a hearth of SDG Loc. 2 cultural layer 2, which indicates the exploitation of bone materials. Grinding of bone artifacts was common in the European Upper Paleolithic period. However, these are rare in China. Previously, the only two sites which yielded bone needles were 


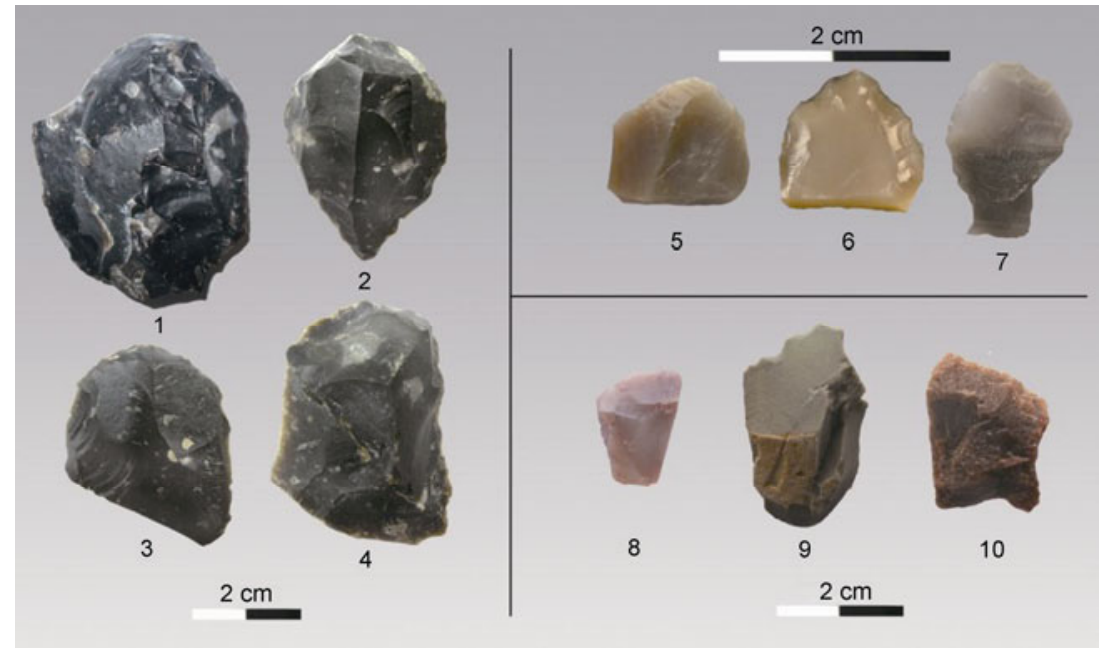

Figure 1 End scrapers and scrapers from SDG Loc. 2.

the Xiaogushan site in Liaoning Province [23,24] and the Upper Cave in Zhoukoudian. The bone needle represents two aspects of ancient human cognition. Firstly, the manufacture flow of bone needle includes the selection of raw materials, interception of bone fragments, and polishing and drilling, which require synthetical techniques of polishing, sawing and drilling [25]. This information suggests that the ancient needle maker had an understanding of the physical properties of raw materials, which implies a high level of rational thinking had been achieved. Secondly, the appearance of bone needles indirectly illustrates the existence of a tailoring medium and the ability to sew objects. The tailoring medium might have involved the use of vegetable fiber or animal hair, while the sewing of objects might have involved animal skin or bark and leaves of plant. Regardless of the medium and the object, the final products were clothes and other objects to maintain warmth. This developed behavior was vital for early modern humans in cold environments, and provided the foundation for their survival and evolution in North China.

(3) Systematic use of body decorations. Ostrich eggshell beads and fragments recovered around earth-pit hearths are major features of CL 2 (Figures 2 and 3), and represent body decoration activities and aesthetic pursuits. This phenomenon has not been documented in the Lower Paleolithic or most Middle Paleolithic sites. Body decoration has been considered an activity that transmits self-awareness, and a mark of individual status and community $[10,26]$. Also, the beads in SDG Loc. 2 are generally dyed, indicating the utilization of pigment in this period. We suggest two possible sources for the pigments: (1) local resources (e.g. hematite) near the camp, which ancient occupants exploited and ground into powder; or (2) in the absence of local pigment resources, these materials were acquired through goods exchange with populations from other areas. We cannot provide conclusive evidence as to whether mineral exploitation or goods exchange existed during the SDG Loc. 2 occupation period. Even so, the sum total of archeological evidence

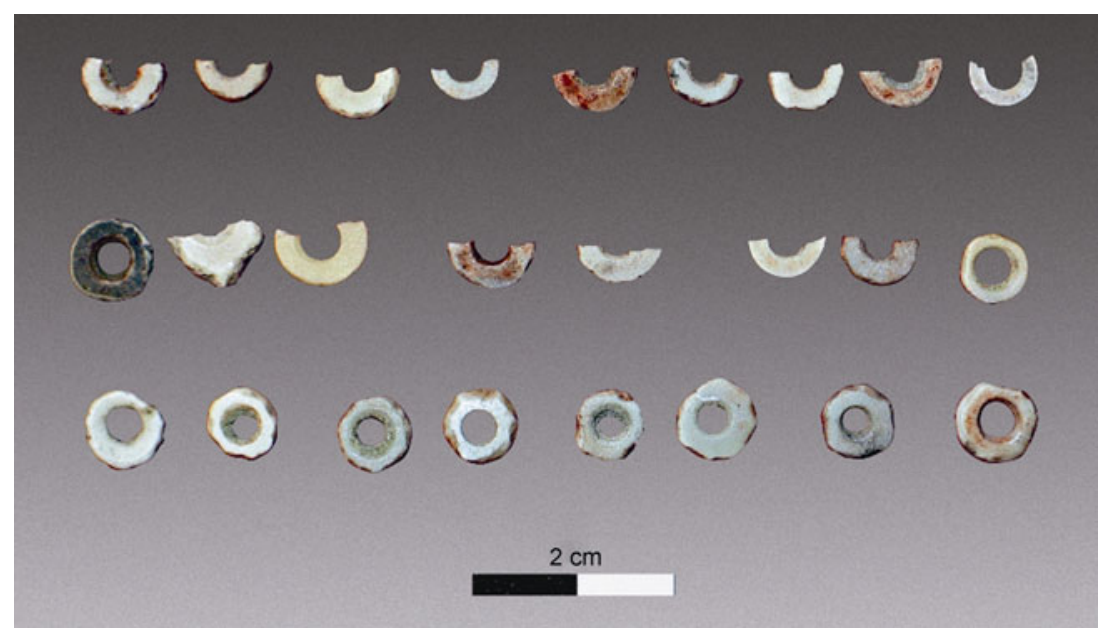

Figure 2 Ostrich eggshell ornaments from SDG Loc. 2. 

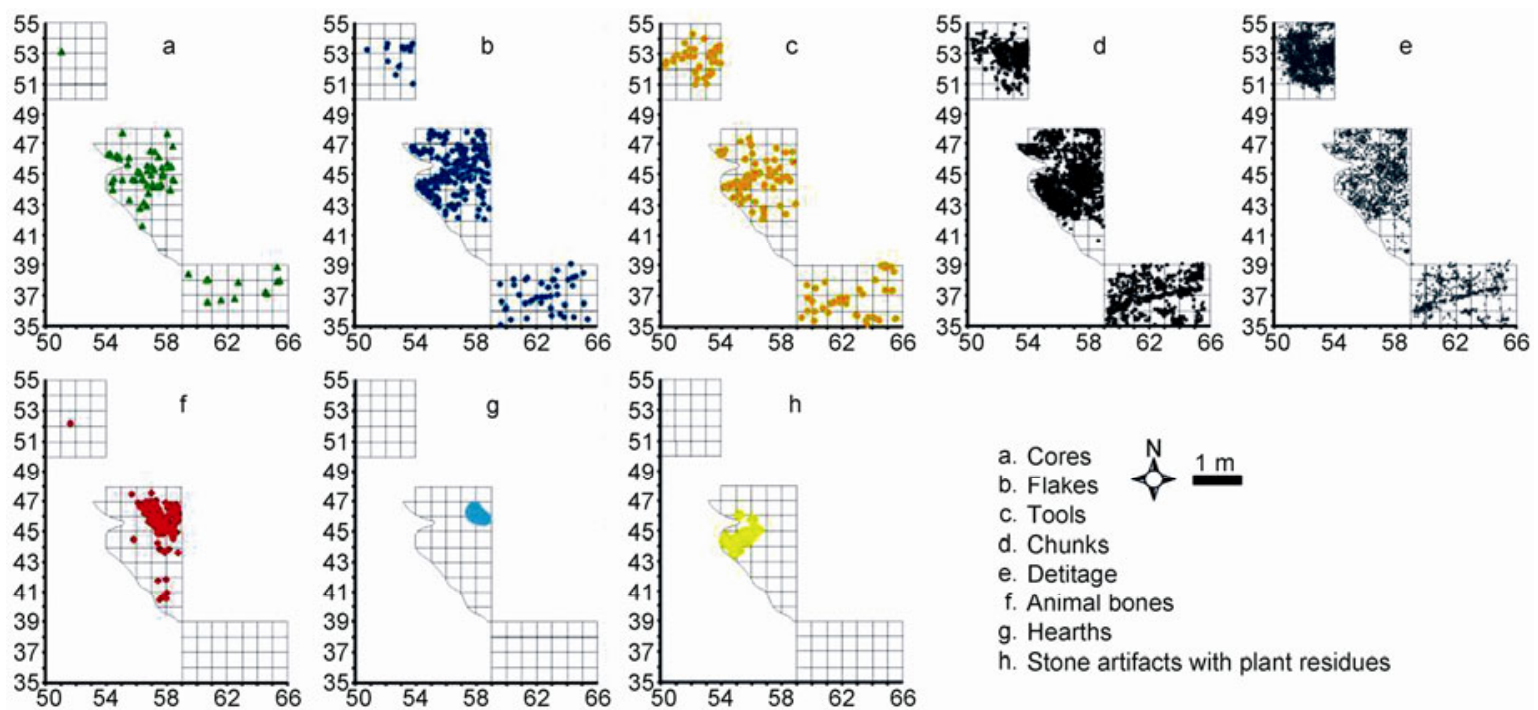

Figure 3 Horizontal distribution of specimens and relics in cultural layer 1 of SDG Loc. 2.

suggests that social structure, human consciousness and behavior had developed to a relatively high level by that time.

(4) Extensive use of earth-pit hearth. Eight earth-pit hearths were recorded on the living floors (area range of 1-4 $\mathrm{m}^{2}$ ) of SDG Loc. 2. These hearths were not constructed by rocks, but appeared as shallow holes in the ground. The utilization of fire can be traced back to the Lower Paleolithic period. However, the systematic and complicated use of fire, which functioned as rock heat treatment, and aided the modification of bone, antler and other materials, was central to the occupants, and was used even for primitive religious events. Such activities only took place in the Upper Paleolithic period, and are considered indicative of early modern human behavior.

(5) Distinct functional spatial organization within habitations. Through the reconstruction of a three-dimensional distribution of cultural remains in cultural layers 1 and 2, the spatial information of different specimens and relics was acquired. Among these, the distribution of stone artifacts in cultural layer 1 is remarkable (Figure 3). Cores and flakes were most abundant around the hearths, especially in the southwest part of the excavation area. Conversely, debitage was found mostly in the north part of the excavation area, far away from the hearths. A possible explanation for this distribution could be that the ancient occupants carried out flaking activities, as well as the re-sharpening and maintenance of stone tools, around the hearths, and then took the flakes to areas far away from the hearths for retouching. The north part of the excavation area may have been such a "retouch and maintenance area". Another possibility is that the north part of the excavation area was a "dumping area". When debitage and broken flakes accumulated in the main activity area, the normal activities of occupants may have been disturbed, thus the need for to establish "trash" areas further way (i.e. northern part), where the highest density of debitage was found.

We also examined the residues from the surface of 103 stone artifacts. More than 30 specimens from cultural layer 1 yielded plant residues, including starch grains, phytoliths and tissue fragments. These stone artifacts with plant residues were mostly distributed to the southwest of the only earth-pit hearth in cultural layer 1 . This discovery demonstrates that ancient occupants did intensive plant processing in this area, which suggests the presence of discrete functional units.

Based on the evidence above, we suggest that in cultural layer 1 of SDG Loc. 2, there were several discrete functional units, including "food processing", "flaking", "retouch and maintenance" and "dumping" areas.

(6) Conversion of subsistence patterns based on utilization of grass seeds. We found abundant starch grains of grass seeds in the plant residues from stone artifact surfaces. Based on comparisons with modern plant collections, 35 individuals (from 9 stone artifacts) were identified to be starch from the seeds of wild Triticeae (Figure 4).

The Triticeae are the well-known cereals, such as Hordeum, Triticum, and Secale. In addition, the genera Aegilops and Agropyron also belong to the Triticeae. The results indicate that the SDG Loc. 2 occupants conducted plant gathering and processing as a subsistence strategy. Also, among these Triticeae starch grains, there were some individuals presenting abnormal damage, amorphous gelation, and loss of their extinction crosses. This damage suggests that the seeds from which the starch grains were derived underwent certain grinding, heating or other processing. Grinding might have occurred through peeling or milling, while heating would have been the result of cooking. It is not likely that this modification would have taken place through the digestive system of humans, since these 


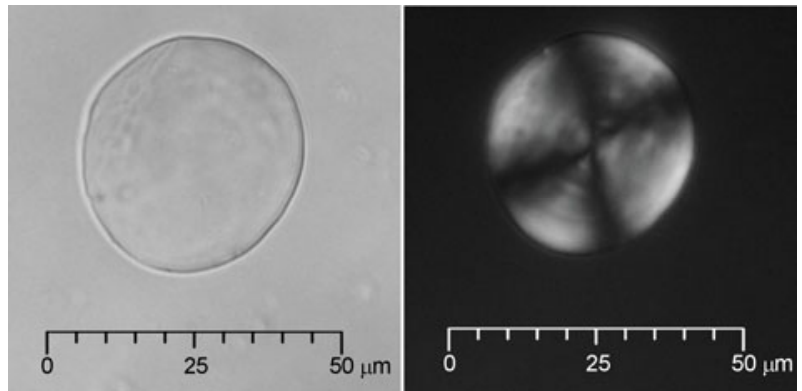

Figure 4 Triticeae starch grain discovered from the surface of a stone artifact of SDG Loc. 2.

materials are not edible as raw cereal starch. Such systematic processing refers to several early modern human behaviors, and provides evidence for plant exploitation by SDG Loc. 2 occupants.

Thus, we deduce that SDG Loc. 2 occupants had already grasped the knowledge of wild cereals gathering, and were aware of the advantages of cereals in terms of nutrition and storability. This knowledge built the cognition foundation of planting activity. In addition, the intensive gathering and consumption of grass seeds indicates the actively broadening diet of humans, and reflects the conversion of subsistence pattern. During the early stage of the Paleolithic period, ancient humans did not gather such high-energy consuming food resources. The mechanism of this change is discussed further below.

In summary, the cultural remains at SDG Loc. 2 represent six typical characteristics of the Upper Paleolithic, and distinctive early modern human behaviors. These characteristics place the SDG Loc. 2 occupants into the Homo sapiens sapiens population, not only from a time scale perspective, but also from a cultural perspective.

\section{Discussion}

\subsection{Characteristics of Homo sapiens sapiens from the "behavior assemblage" perspective}

During human evolution, as with the Mosaic Phenomenon of the physical features [27], cultural factors also alternated between both primitive and advanced elements. This means that certain early modern human behavior factors may have emerged before the origin of Homo sapiens sapiens. Thus, single and accidental advanced behavioral signals should not be considered as reliable indicators of any potential early modern human population. Conversely, a "behavior assemblage" must constitute several reliable behavioral elements to be considered a stable, dependable and reliable indicator.

The conversion of subsistence patterns is one of the characteristics of Upper Paleolithic culture. Rapid diversification in hunting, food processing technologies, social group organization, and the invention of food storage equipment generally accompanied this conversion. Thus, we con- sider all these elements as a "behavior assemblage", and analyze the motivation and mechanism of its occurrence, and subsequently discuss the overall characteristics of early modern humans.

Currently studies of archaeological sites in West Asia and Europe provide abundant evidence to develop theoretical frameworks and hypotheses. However, in East Asia, there is a lack of understanding regarding the mechanisms and patterns of plant resource exploitation during the Paleolithic period. Thus, the history of wild plant use in dry land of North China is an important scientific undertaking.

The gathering and processing of grass seeds in SDG Loc. 2 demonstrates that ancient occupants have already expanded their food resources during the 30-20 ka BP interval, and had shifted gathering targets from low-energy consuming but high-energy returning objects to high-energy consuming and returning objects. Combined with evidence of functional spatial organization, we suggest that the BSR occurred in the SDG area during the SDG Loc. 2 occupational period, and subsequently affected wild plant exploitation patterns in adjacent area.

\section{2 "Broad Spectrum Revolution" and prehistoric plant resources exploitation}

The exploitation of plants is an involved procedure which is both complex and systematic. During the Lower Paleolithic period, ancient people gathered randomly. Thus far, there is no evidence or clues of organized gathering activities during this earlier stage. It is still not clear at what point in time of humans grasped the knowledge of food resource geographic distribution and plant growth rhythm. There are speculations made from the gathering objects used by European Middle Paleolithic populations, which suggest that ancient foragers had focused on underground storage organs (USO hereafter) by this time, such as tubers and roots [28]. With regard to the Upper Paleolithic, abundant archaeological evidence suggests that ancient humans not only retained knowledge of plant nutritional value and seasonality, and the gradual enhancement of processing and storage technologies, but also expanded their diet with the double pressures of climatic change and population expansion in the last glacial. Thus, high-energy consuming resources (e.g. grass seeds) began to be incorporated into their daily diets, and eventually increased in ratio. At some point, planting activities began, although at a low scale and amount, and without organization and regularity.

BRS theory also predicts that in areas with insufficient resources, people were compelled to adopt the planting strategy for survival $[11,12,29]$, and that real cultivation began to dominate in the Holocene (Figure 5).

The Levant region in West Asia provides an example during the last stage of the Pleistocene, when prehistoric populations relied on wild plants, including wild cereals (e.g. wild wheat, wild barley and wild rye), nuts and wild legumes 


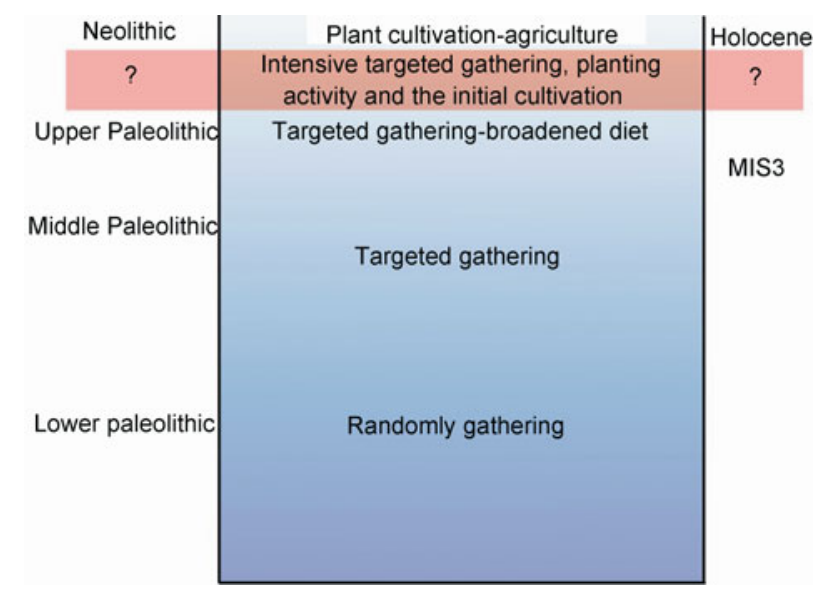

Figure 5 The dynamic procedure of plant use in prehistory.

[29]. These wild species gradually evolved to be the local domesticated crops. Recent archaeological evidence suggests that exploitation of these resources occurred before the last glacial maximum [14-16,30].

\subsection{A human behavioral ecology theory perspective on the study data}

Human behavioral ecology (HBE hereafter) theory developed from the concept of foraging behavioral ecology in biology. In HBE, the "optimization" theorem is the most important premise $[31,32]$. Targeted gathering and planting activities are thought to have resulted from the development of plant gathering behavior. In addition, these activities also are under the control of the optimization theorem.

In terms of SDG Loc. 2, the existence of Triticeae plants seeds represents the broadening of the local human diet. These seeds need much processing before serving. Processing may include peeling and heating to make the starch grains gelatinous and therefore fit for the human digestive system, which requires more time and energy. Thus, in an ideal situation, foragers would not select this resource. Thus, the expansion of gathering objects would be due to other reasons, and would follow certain regulations. We used the Diet Breadth Model (DBM, or Optimal Diet Model) to examine the motivation and mechanism of BSR at the SDG Loc. 2 study site.

In DBM, there are several factors that could have affected foraging strategy organization: total quantity of resources, energy rate, energy input and foraging time. The higher quantity of resources in the resource patch translates into a shorter searching time per item. However, the total handling time always increases. Thus, the "optimization point", or optimal foraging time point, would depend on the quantity the foragers gathered (Figure 6). DBM assumes that gatherers would select the resources with the highest energy return rate $[31,33]$. In other words, all resources would be ranked

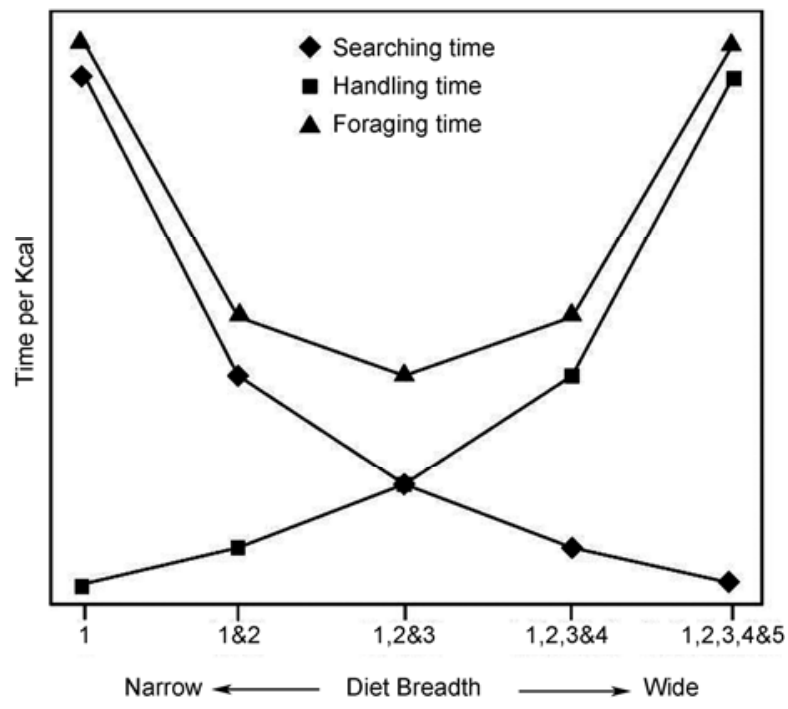

Figure 6 The Diet Breadth Model [34].

by their energy return rates in the same resource patch, and gatherers would prefer resources with high energy return rate, which are easier to handle, instead of gathering all visible resources.

Thus, SDG Loc. 2 occupants may have ranked all the available local resources based on energy return rates, which can be calculated by the following formula:

\section{Kilocalorie/Gram $\times$ Gram $\div$ Hour.}

In this formula, "Kilocalorie/Gram" means the energy rate; "Gram" means the quantity of the acquired resources; "Hour" represents the foraging time, including the searching time $(S)$ and the handling time $(H)$. This means that $S$ and $H$ are two important parameters of gathering activities. In terms of $S$, the most direct influencing factor is the geographic distribution of resources. In another words, if the resource density is high and easy to search, the searching time will be decreased, so that foraging time also decreases. Thus, the energy return rate increases. Conversely, if distribution is sparse, requiring a longer search time, the foraging time increases, and the energy return rate decreases [32] ${ }^{1}$. In terms of $H$, it will be considerably affected by the technological level of the foragers. For example, the use of a sickle will enhance efficiency compared with unarmed gathering [32].

The SDG Loc. 2 site was occupied during 30-20 ka BP, and presented no development of stone tools technology and morphology, which indicates that all cultural layers belong to the same Paleolithic tradition. Thus, we exclude the possibility of technological transformation and consider the "handling time" constant invariable. In this situation, when the plant energy rate does not change, the only parameter to be considered is the "searching time", which is the geographic distribution condition of different plants.

1) In this historical period, horse riding and other transport enormously affected the search time. However, this factor could be ignored for the Paleolithic age. 
According to ethnographic data [34-38], the Post-encounter Return rate (when $S=0$, the energy return rate, also called the Post-encounter Return rate: Kilocalorie/Gram $\times$ Gram $\div$ Hour) of 5 types with 30 species of plants are shown in this table (Table 1).

This means that in ideal circumstances, the foragers would have selected the resources based on energy rates. However, in the last interglacial, the climate fluctuated dramatically, temperature and precipitation varied each year in the SDG area. These conditions caused the decrease and extinction of many plant species. As a result, the density of many species was reduced, which increased searching time, and decreased the energy return rate. Searching for nuts became considerably affected. The decrease or fluctuation of precipitation also caused a decrease of broad-leaf and coniferous trees. In addition, the SDG area was basically a grass-forest open environment, in which there were few trees. With decrease in the number of these trees, the search for nuts became increasingly difficult. In this situation, nuts could not be considered a stable food resource. With regards to USOs, they also were relatively difficult to find, since they were buried underground, and therefore also might have been scarcer in the fluctuating climate. In this case, Poaceae plants had many advantages, including being more tolerant to environmental conditions, highly drought resistance, widely distributed, non-toxic, and producing starchy seeds. As the energy return rates of nuts and USOs decreased, the seeds of Poaceae plants gradually became the favorites of foragers.

The entire dynamic process is shown in Figure 7. With the same searching time, the USO had the highest energy return rate, followed by nuts, and then cereal. When impacted by environmental factors, USO and nut became scare, causing the expansion of search time, and a gradual drop in energy return rate. However, the distribution of cereal stayed the same because of adaptations to the changing environment. Moreover, the searching time for cereal almost remained unchanged. When the searching time arrived at S1 in Figure 7, the energy return rates of USO and cereal achieved the same level. When the searching time reached $\mathrm{S} 2$, the energy return rates of nut and cereal achieved the same level. Finally when the searching time continued to grow, the energy return rate of cereal became the highest.

\section{Conclusions}

We make the following conclusions about the survival strate-

Table 1 Post-encounter Return rate ${ }^{\text {a) }}$

\begin{tabular}{cccccc}
\hline \multicolumn{1}{c}{ Type } & USO & Nuts & Legumes & Cereals & Fruits \\
\hline $\begin{array}{l}\text { Post-encounter } \\
\text { Return rate }\end{array}$ & 1853.11 & 1337.43 & 989.5 & 697.83 & 250 \\
\hline
\end{tabular}

a) Based on 30 types of high latitude region plant resources.

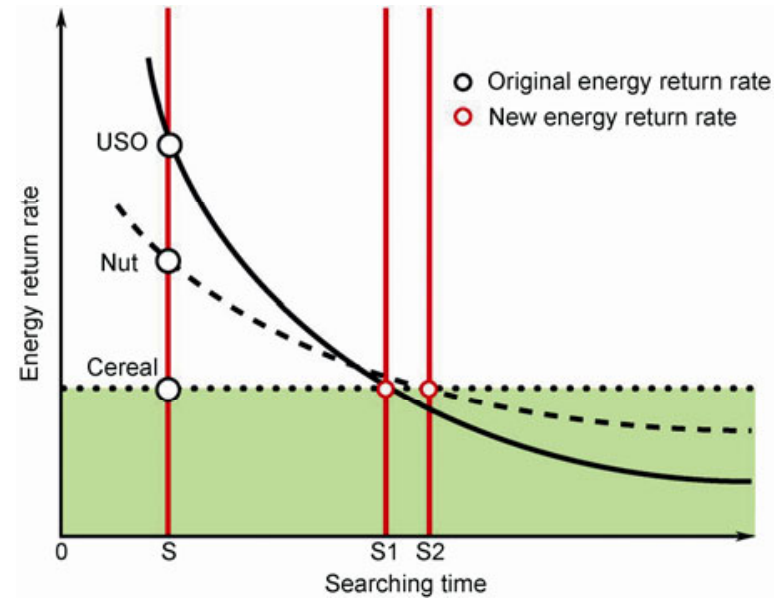

Figure 7 Resources energy rate of return change dynamic process.

gies and behaviors of SDG Loc. 2 occupants. First, as a base camp, SDG Loc. 2 served as a center of human activities, including stone knapping, bone tools manufacture, ornament making, and production of clothes or other warmth items. The camp was used comprehensively with several discrete functional units. With the increasing pressure of environmental change or population growth, more resources were selected as food materials, thus causing distinct survival pattern changes. SDG Loc. 2 occupants are the oldest early modern human populations which can be confirmed from the cultural and behavioral perspectives.

Ancient human cognition developed with the evolution of intellect. At some point in the Paleolithic period, ancient humans began to grasp knowledge about the different energy rates of various plants, and consequently focused on USOs, or high energy resources such as nuts. Gradually, when the geographic distribution and density of these resources changed, ancient humans expanded their diet based on energy return rates. In other words, when the energyload level decreased in the local environment, the broadening diet was an active response and strategy by ancient foragers to the living environment.

Wild cereals gathering and processing were strongly associated with the emergence of primitive agriculture. The time period and patterns of dry land agriculture emergence is still a topic of heated debate, and the discovery of Triticeae starch grains from SDG Loc. 2 sheds new insights on this debate. The "Broad Spectrum Revolution" was not a short time event, but a long process which may have taken tens of thousands of years to accomplish. BSR occurred not only in West and East Asia, but also in Europe, America, Australia and Africa, and accelerated the exploitation of wild plants by prehistoric populations, and finally brought about planting activities on the world stage.

Wang Huimin, Zhong Kan, Feng Xingwu, Zhang Shuangquan, Luo Zhigang, Zhang Yue, Zhang Xiaoling et al. made significant contributions to the field work, and the authors express deep thanks to them. This work was 
supported by the Strategic Priority Research Program of the Chinese Academy of Sciences (XDA05130303), the Knowledge Innovation Program of the Chinese Academy of Sciences (KZCX2-YW-Q1-04), and the National Basic Research Program of China (2010CB950203).

1 Gao X, Zhang X, Yang D, et al. Revisiting the origin of modern humans in China and its implications for global human evolution. Sci China Earth Sci, 2010, 53: 1927-1940

2 Dennell R. The Palaeolithic Settlement of Asia. Cambridge: Cambridge University Press, 2009

3 Straus L G, Eriksen B V, Erlandson J M, et al. Human at the end of the Ice Age. In: The Archaeology of the Pleistocene Holocene Transition. New York: Plenum, 1996

4 Wolpoff M, Wu X, Thorne A. Modern homo sapiens origins: A general theory of hominid evolution involving the fossil evidence from East Asia. In: Smith F, Spencer F, eds. The Origin of Modern Humans. New York: Alan R Liss Inc, 1984. 411-483

5 Zhang S. Reginal industrial gradual advance and cultural exchange of Paleolithic in North China (in Chinese). Acta Anthropol Sin, 1990, 9: 322-333

6 Zhang S. Paleolithic Culture in China (in Chinese). Tianjin: Tianjin Science and Technology Press, 1987

7 Zhang S. On the important advancements of the Paleolithic archaeology in China since 1949 (in Chinese). Acta Anthropol Sin, 1999, 18: 193-214

8 Gao X, Pei S. An archaeological interpretation of ancient human technology and adaptive strategiesin China (in Chinese). Quat Sci, 2006, 26: 504-513

9 Bar-Yosef O. The archaeological framework of the Upper Paleolithic Revolution. Diogenes, 2007, 54: 3-18

10 Bar-Yosef O. The Upper Paleolithic revolution. Rev Anthropol, 2002, 31: 363-393

11 Flannery K V. Origins and ecological effects of early domestication in Iran and the Near East. In: Ucko P J, Dimbleby G W, eds. The Domestication and Exploitation of Plants and Animals. Chicago: Aldine Publishing Company, 1969. 73-100

12 Binford L R. Archaeological perspectives. In: Binford S R, ed. New Perspectives in Archaeology. Chicago: Aldine Publishing Company, 1968. 5-32

13 Stiner M C. Thirty years on the "Broad Spectrum Revolution" and paleolithic demography. Proc Natl Acad Sci USA, 2001, 98: 6993-6996

14 Weiss E, Wetterstrom W, Nadel D, et al. The broad spectrum revisited: Evidence from plant remains. Proc Natl Acad Sci USA, 2004, 101: 9551-9555

15 Piperno D R, Weiss E, Holst I, et al. Processing of wild cereal grains in the Upper Palaeolithic revealed by starch grain analysis. Nature, 2004, 430: 670-673

16 Weiss E, Kislev M E, Simchoni O, et al. Small-Grained wild grasses as staple food at the 23000-year-old site of Ohalo II, Israel. Econ Bot, 2004, 58: S125-S134

17 Ningxia Museum and Ningxia Geological Survey. Excavation of the Shuidonggou site in 1980 (in Chinese). Acta Anthropol Sin, 6: 439-448

18 Ningxia Archaeological Institute. Shuidonggou-1980 Excavation
Report (in Chinese). Beijing: Science Press, 2003

19 Zhou K, Hu J. Environment and stratigraphy at the Shuidonggou site (in Chinese). Acta Anthropol Sin, 1988, 7: 263-269

20 Liu D, Wang X, Gao X, et al. Progress in the stratigraphy and geochronology of the Shuidonggou site, Ningxia, North China. Chin Sci Bull, 2009, 54: 3880-3886

21 Gao X, Yuan B, Pei S, et al. Analysis of sedimentary-geomorphologic variation and the living environment of hominids at the Shuidonggou Paleolithic site. Chin Sci Bull, 2008, 53: 2025-2032

22 Gao X, Li J, Madsen D B, et al. New ${ }^{14} \mathrm{C}$ dates for Shuidonggou and related discussions (in Chinese). Acta Anthropol Sin, 2002, 21: 211218

23 Zhang Z, Fu R, Chen B, et al. A preliminary report on the excavation of Paleolithic site at Xiaogushan of Haicheng, Liaoning Province (in Chinese). Acta Anthropol Sin, 1985, 4: 70-79

24 Huang W, Zhang Z, Fu R, et al. Bone artifacts and ornaments from Xiaogushan site of Haicheng, Liaoning Province (in Chinese). Acta Anthropol Sin, 1986, 5: 259-266

25 Huang Y. A study of manufacture and use of bone needle from Xiaogushan (in Chinese). Archaeology, 1993, 3: 260-268

26 Wang C, Zhang Y, Gao X, et al. Archaeological study of ostrich eggshell beads collected from SDG site. Chin Sci Bull, 2009, 54: 3887-3895

$27 \mathrm{Wu}$ X. The evolution of humankind in China (in Chinese). Acta Anthropol Sin, 1990, 9: 312-321

28 Hardy B L. Climatic variability and plant food distribution in Pleistocene Europe: Implications for Neanderthal diet and subsistence. Quat Sci Rev, 2010, 29: 662-679

29 Flannery K V. The origins of agriculture. Rev Anthropol, 1973, 2: 271-310

30 Weiss E, Kislev M E, Simchoni O, et al. Plant-food preparation area on an Upper Paleolithic brush hut floor at Ohalo II, Israel. J Archaeol Sci, 2008, 35: 2400-2414

31 Shennan S. Genes, Memes and Human History: Darwinian Archaeology and Cultural Evolution. London: Thames and Hudson, 2002

32 Kelly L R. The Foraging Spectrum: Diversity in Hunter-Gatherer Lifeways. Washington D C: Smithsonian Institution Press, 1995

33 Bettinger R L. Hunter-Gatherers: Archaeological and Evolutionary Theory. New York: Plenum Press, 1991

34 Cane S. Australian aboriginal subsistence in the Western Desert. Human Ecol, 1987, 15: 391-434

35 O'Connell J F, Hawkes K. Alyawara plant use and optimal foraging theory. In: Winterhalder B, Smith E A, eds. Hunter-Gatherer Foraging Strategies. Chicago: University of Chicago Press, 1981. 99-125

36 Simms S. Behavioral Ecology and Hunter-Gatherer Foraging: An Example from the Great Basin. Oxford: British Archaeological Reports, International Series 381, 1987

37 Couture M D, Ricks M F, Housley L. Foraging behavior of a contemporary Northern Great Basin population. J California Great Basin Anthropol, 1986, 8: 150-160

38 Winterhalder B. Foraging strategies in the Boreal Forest: An analysis of Cree hunting and gathering. In: Winterhalder B, Smith E A, eds. Hunter-Gatherer Foraging Strategies. Chicago: University of Chicago Press, 1981. 66-98

Open Access This article is distributed under the terms of the Creative Commons Attribution License which permits any use, distribution, and reproduction in any medium, provided the original author(s) and source are credited. 